Preprints of the

Max Planck Institute for

Research on Collective Goods

Bonn 2012/12

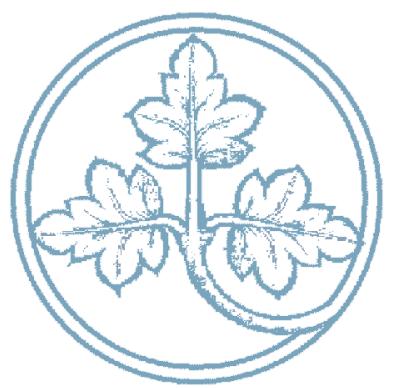

The Role of Information in Different Bargaining Protocols

Rafael Hortala-Vallve Aniol Llorente-Saguer Rosemarie Nagel

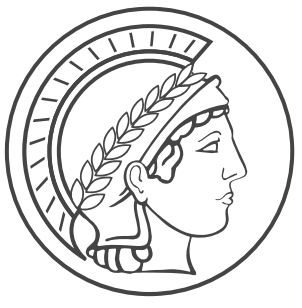




\section{The Role of Information in Different Bargaining Protocols}

Rafael Hortala-Vallve / Aniol Llorente-Saguer / Rosemarie Nagel

May 2012 


\title{
The Role of Information in
}

\section{Different Bargaining Protocols}

\author{
Rafael Hortala-Vallve \\ Government Department \\ London School of Economics
}

\author{
Aniol Llorente-Saguer \\ Max Planck Institute for \\ Research on Collective \\ Goods, Bonn
}

\author{
Rosemarie Nagel \\ Universitat Pompeu Fabra \\ ICREA
}

May 2012

\begin{abstract}
We analyze a bargaining protocol recently proposed in the literature vis-à-vis unconstrained negotiation. This new mechanism extracts "gains from trade" inherent in the differing valuation of two parties towards various issues where conflict exists. We assess the role of incomplete vs. complete information in the efficiency achieved by this new mechanism and by unconstrained negotiation. We find that unconstrained negotiation does best under a situation of complete information where the valuations of both bargaining parties are common knowledge. Instead, the newly proposed mechanism does best in a situation with incomplete information. The sources of inefficiencies in each of the two cases arise from the different strategic use of the available information.
\end{abstract}

\footnotetext{
We thank Antonio Cabrales, Alessandra Casella, Gary Charness, Rachel Croson, Andy Eggers, Christoph Engel, Ido Erev, Guillaume Frechette, Simon Hix, Robin Hogarth, Marco Kleine, Matthias Lang, Nagore Iriberri, Tom Palfrey, Andy Schotter and seminar participants at a number of conferences for helpful comments and discussions. We specially want to thank two anonymous referees for encouraging us analyzing the role of information in the previously circulated paper "Experimental Comparison between Free Negotiation and a Multi-issue Point Mechanism”. We would also like to thank Benedikt Herz, Pablo Lopez Aguilar Beltran and Gwendolin Sajons for excellent assistance at running the experiments. The first author acknowledges financial support from the British Academy; the third author acknowledges financials support from ECO2008-01768 and the Generalitat de Catalunya and the CREA program.
} 


\section{Introduction}

Negotiations between two parties are an important recurring element in our lives: buyer and seller, political parties, international disputes, bilateral agreements in arms/pollution reduction, etc. The process, outcome and success of negotiations depend on the characteristics of the setup and the information available to the bargaining parties.

One of the key characteristics in any negotiation is the number of items over which disagreement exists. Whenever negotiations occur in a single dimension (such as setting the price in a buyer/seller transaction) gains for one party are losses for the other party. Instead, negotiations with multiple dimensions allow strict Pareto improvements. In such situations, parties need to "assess tradeoffs in terms of how much to give up in [...] one issue in order to obtain a specified gain in another issue”" Precisely, a central aspect in the negotiation literature is whether negotiating parties can exploit the gains of trade inherent in their different valuation of the issues (see for instance Fisher and Ury, and Pattan, 1981, Bazerman and Neale, 1983, Keeney and Raiffa, 1991, or Thompson, 2000).

Motivated by the same idea, there is burgeoning literature on mechanisms that allow bargaining parties to trade off their intensities of preferences in order to influence the final outcome on several issues. ${ }^{2}$ At the heart of these new mechanisms is the idea that by linking decisions, relative intensities across issues can be elicited and strict Pareto improvements can be made possible.

The goal of this paper is to relate these new mechanisms to the way we usually resolve our disputes, i.e. unconstrained negotiation. Most importantly we want to assess the role of information in the efficiency achieved by these different bargaining protocols. We design a set of experiments and compare the outcomes reached by unconstrained negotiation to those obtained with a point mechanism where two conflicting parties can unilaterally trade off their influence across the various issues at stake. We replicate the same situations under both bargaining protocols in order to compare and evaluate the advantages of each mechanism. We vary the information available to subjects: when information is complete, the preferences (payments) of both bargaining parties are common knowledge; when information is incomplete bargaining parties only know their own preferences (payments) for each issue.

Our point mechanism is essentially the same as the one proposed by Brams and Taylor (1996), Casella (2005, 2011), and Hortala-Vallve (forthcoming): each of the two bargaining parties is endowed with a budget of points that need to be distributed across a set of issues simultaneously. Conflicting parties have opposing preferences so that one party wants all issues approved and the opposing party wants them all dismissed. The party that invests most votes on a given issue is decisive on that issue. In the case of a tie, the issue is approved or dismissed with equal probability. By unilaterally trading off bargaining power from less preferred issues to

\footnotetext{
${ }^{1}$ Keeney and Raiffa (1991)

${ }^{2}$ See for instance Brams and Taylor (1996 and 1999), Casella (2005, 2011), Jackson and Sonnenschein (2007), and Hortala-Vallve (forthcoming).
} 
more preferred ones, parties can extract the gains from trade inherent in their differing relative intensities. We compare this mechanism to a situation of unconstrained negotiation where subjects can communicate in writing (via computer terminals) and can propose final allocations.

We find that improving the available information to bargaining parties has opposite effects under our two bargaining protocols. Under unconstrained negotiation, subjects reach a higher average welfare when preferences are common knowledge rather when information is incomplete. Instead, when using the point mechanism, average welfare is superior when subjects only know their own preferences as compared to a situation with complete information. Comparing the two protocols under the same information condition, we find that negotiation is superior over the point mechanism under full information and the reverse is true under incomplete information. In section 5 we discuss the likely sources of these differences which lie in the strategic use of the private information. When subjects cast their points simultaneously, as in the point mechanism, it is beneficial for the overall welfare that subjects do not know their opponent's preferences: in that case subjects are prone to play monotone strategies -in the sense that more points are invested in the issues that are more valued- and this strategy profile reaps most efficiency gains. Instead, when bargaining parties have complete information about preferences, they have incentives to strategize and invest their points so as to increase the probability of winning most of the issues. When subjects freely negotiate under a situation of incomplete information they strategize (or bluff): there is an incentive to misrepresent one's preferences and this strategic use of privately held information yields Pareto inefficient situations. Instead, such inefficiencies are more likely to be avoided when information is complete.

Our work shows the appealing properties of the novel mechanisms that link issues such as the Adjusted Winner, Storable Votes or Qualitative Votes, vis-à-vis unconstrained negotiation. When information is incomplete, these mechanisms allow bargaining parties to simultaneously trade off their influence across multiple issues and reach a final allocation that is superior to the one reached through unconstrained negotiation. These mechanisms mimic the heuristics that lead to good agreements by inducing integrative bargaining across the various dimensions: they force parties to consider all issues simultaneously ${ }^{3}$ and, given the lack of information, induce monotone strategies thus allowing bargaining parties to be decisive on those issues they feel strongly about.

The paper is structured as follows: the remaining part of this section presents the related literature; Section 2 explains in detail the two bargaining protocols we consider. Section 3 describes the experimental design and Section 4 presents our main results: the point mechanism does best with incomplete information while unconstrained negotiation does best with complete information. Finally, Section 5 discusses the likely source of our main result and Section 6 concludes.

\footnotetext{
${ }^{3}$ Considering issues simultaneously is one of the keys for reaching integrative bargaining. See for instance Erickson et al (1974), Kelley (1966), Pruitt (1981) or Yukl et al (1976).
} 


\subsection{Related literature}

The literature on negotiating practices spans several decades and methodologies. Important contributions have been summarized in a number of textbooks (see for instance, Bazerman \& Neale, 1992; Fisher \& Ury, 1981; Murnighan, 1991; Raiffa, 1982; Thompson, 2000). However, to the best of our knowledge there are no comparisons between unconstrained negotiation and mechanisms that may improve the allocation for both parties. There is, however, some theoretical literature that studies software designed to support various negotiation activities, called negotiation support systems (see Bui, 1994; Holsapple and Whinston, 1996; Kilgour, 1996). One could view our point mechanism as one such negotiation support system.

There is also a large literature from experimental economics and psychology that studies negotiating (or bargaining) practices. The majority of this experimental literature draws on the ultimatum game where one party decides his share of a prize but needs the approval of the recipient for the remainder of the prize (see the seminal paper Guth et al, 1982). The role of one-sided asymmetric information has been studied by, e.g., Mitzkewitz and Nagel (1993) and Croson (1996). These papers show that subjects use privately held information to their advantage. This is in line with earlier work by Roth and Murnighan (1982) who compare bargaining under complete and incomplete information and show how bargaining outcomes are significantly different when the level of information changes. Excellent surveys on bargaining can be found in Roth (1995) or Camerer (2003).

Departing from this work, there is a growing literature that looks at multidimensional bargaining situations and that, in particular, tests the mechanisms referred to in the introduction. Daniel and Parco (2005) and Schneider and Kramer (2004) test the adjusted winner, proposed in Brams and Taylor (1996, 1999); Casella et al (2006, 2008) test the storable votes proposed in Casella (2005, 2011); Engelmann and Grimm (forthcoming) test the linking mechanism proposed in Jackson and Sonnenschein (2007); and Hortala-Vallve and Llorente-Saguer (2010) test the PM proposed in Hortala-Vallve (forthcoming). Except for the experiments on the adjusted winner - which lack a game-theoretical prediction - these papers conclude that although subjects depart from theoretical behavior, welfare levels are remarkably close to theoretical predictions. All these mechanisms have been studied in environments with incomplete information. So far there have been no papers that compare the role of information on the efficiency reached by these mechanisms. This paper will be only a first step to fill this gap. 


\section{Point mechanism and negotiation}

Two players have opposing views over $N(N \geq 2)$ issues that need to be approved or dismissed. Monetary transfers are not allowed. Players and issues are denoted $i \in\{1,2\}$ and $n \in\{1,2, \ldots, N\}$, respectively. Player $i$ 's valuation towards issue $\mathrm{n}$ is denoted $\theta_{i}^{n} \in \mathfrak{R}^{+}$. The preference vector of player $i$ is denoted $\theta_{i}=\left(\theta_{i}^{1}, \ldots, \theta_{i}^{N}\right)$ and his payoff on issue $\mathrm{n}$ is $\theta_{i}^{n}$ if his will is implemented in issue $\mathrm{n}$ and 0 if his opponent's will is implemented in issue $\mathrm{n}$.

The total payoff is the sum of the individual payoffs across the $N$ issues. Therefore, we are implicitly assuming the lack of complementarities between issues (e.g. we do not allow for the valuation of an issue to depend on the approval or dismissal of a different issue). While we need this assumption for our result to hold, we can consider any linear transformation of the payoffs. In particular, in our experimental setup we normalize payments so that the sum of valuations across dimensions is constant for each individual.

Without loss of generality we assume that player $i$ wishes the approval of all issues and player $j$ wishes their dismissal. Throughout we keep the interpretation that players are deciding simultaneously over the approval or dismissal of various issues. However, our model could also be interpreted as a situation where two players are auctioning $N$ indivisible objects.

We analyze two bargaining protocols. In the first, players are endowed with $P$ indivisible points that can be freely distributed between the issues. The number of points is fixed and independent of the number of issues. The action space is the collection of voting profiles:

$$
\left\{\left(p^{1}, \ldots, p^{N}\right) \in\{0,1, \ldots, P\}^{N}: p^{1}+\ldots+p^{N}=P\right\} .
$$

Hereafter we call this mechanism the point mechanism. This mechanism allows each player to implement his will on issues where he invests more points than his opponent. Ties are broken with the toss of a fair coin. That is, when $p_{i}^{n}>p_{j}^{n}$ player $i$ decides on issue $\mathrm{n}$ and when $p_{i}^{n}=p_{j}^{n}$ each player decides on issue $\mathrm{n}$ with equal probability. ${ }^{4}$ This mechanism is the one proposed in Hortala-Vallve (forthcoming). It also resembles other mechanisms in which players can distribute points (or votes) across issues such as the Adjusted Winner (Brams and Taylor 1996, 1999) or Storable votes (Casella 2005, 2011).

Our second bargaining protocol is unconstrained negotiation. Players can communicate (cheap talk) and need to reach an unanimous decision on whether each issue is approved, dismissed or tied. For obvious time constraints in our experimental setup we impose that if such an agreement is not reached in a predetermined amount of time, a status quo outcome (where all issues are tied) is implemented.

\footnotetext{
${ }^{4}$ For simplicity, in our experiment subjects earn half their valuation whenever ties occur on an issue. That is, we do not decide on a tied issue with the toss of a fair coin; instead we assign the expected value of such randomisation.
} 


\section{Experimental design and Procedures}

In our experimental sessions and treatments, subjects repeatedly played several bargaining games. In each period subjects were randomly matched into pairs and the two players had to decide on the outcome of three issues -issues could be tied or 'won' by either of the matched subjects. ${ }^{5}$ We assigned four treatments according to two different criteria.

The first criterion of the design is the bargaining protocol. In each session subjects were confronted with only one of the protocols, the Point Mechanism (PM) or Negotiation (NEG). In the PM sessions, the number of points to be distributed by each subject was six; subjects had no time constraint (they needed about 20 seconds in average across different treatments). In the future it will be interesting to analyze the role of the number of points each subject has at its disposal. It remains however an open question what would be the optimal strategy in such circumstance. ${ }^{6}$ For this reason we prefer focusing our analysis to a situation in which we know the equilibrium actions and thus the subjects' behavior can be analyzed vis-à-vis our theoretical predictions. ${ }^{7}$

In the NEG sessions, subjects could freely negotiate through a chat box for four minutes. This communication was unrestricted with the only exception that subjects could not reveal their identity. ${ }^{8}$ Parallel to their communication, subjects could send binding proposals to the subject they were matched with, specifying the outcome in each of the three issues. The period would end when a player accepted the opponent's proposal. If no proposal was accepted within the four minutes time limit, all issues were drawn and subjects proceeded to the next period. ${ }^{9}$

The second criterion of the design was the informational setting. In each session subjects were exposed to only one setting: either they received complete information about their own preferences and the preferences of the

\footnotetext{
${ }^{5}$ We partitioned the subjects into three sets of six players (or two sets of ten players) so as to obtain three (two) independent observations. Throughout the paper we present non-parametric tests using the average of each independent group as a single data point.

${ }^{6}$ Whenever information about preferences of the other player is incomplete (which will be the case for one of the treatments), the game played is a non-zero sum Colonel Blotto game with incomplete information. A Colonel Blotto Game can be described by a situation where two colonels are fighting over a number of regions and have to decide how to divide their forces; the one with larger forces wins the region and the winner of the battle is the one who wins the most territory. This is, unlike in our setting, a zero-sum game because both colonels value all regions equally. Recently, Robertson (2006) and Hart (2006) have provided a complete solution of the Colonel Blotto game when parties value all issues equally, but the game has yet to be solved in our case where opposing parties have differing relative intensities (so there are gains to be extracted)

${ }^{7}$ A fully fletched analysis of individual behaviour under the PM can be found in the Hortala-Vallve and LlorenteSaguer (2010) -a short summary of this paper's key findings is included in the appendix to this paper.

${ }^{8}$ In the instructions we stated that revealing one's identity implied being excluded from the lab's recruiting list; we did not find anyone breaking this rule.

${ }^{9}$ Carnevale and Lawler (1986) claim that most negotiations occur under some form of time pressure. Besides it is not feasible to run a laboratory experiment without such constraints as the possibility of gridlock may freeze the experiment indefinitely. Yukl et al (1976) and Carnevale and Lawlwer (1986) show that early deadlines can affect negatively the payoff of integrative negotiations. Roth, Murnighan, and Schoumaker (1988) show that tied deadlines lead to delaying bargaining into the last seconds. We tried avoiding such effects by running a pilot experiment and implementing a time constraint above the maximum time subjects declared optimal.
} 
subject they were matched with, or there was incomplete information whereby subjects only knew their own preferences and the distribution from which their opponent's preferences were drawn. In all situations preferences were induced by assigning a valuation in Eurocents to each of the issues. In each period, valuations were randomly drawn from a uniform distribution of vectors with elements being positive multiples of 50; no issue was valued zero, and the valuations in each vector summed to $600 .{ }^{10}$ The purpose of the constant total valuation was twofold. Firstly, it ensured comparability across games and avoided framing effects. ${ }^{11}$ Secondly, all subjects are weighed equally when we later calculate the efficient utilitarian outcome (without having to weigh each observation appropriately).

In our experimental sessions we avoided any reference to the issue being approved or dismissed because we wanted both matched subjects to be in a symmetrical situation and we feared that labeling the outcome of each issue in terms of approval or dismissal may have an effect. Various studies show that negotiations usually reach a more efficient allocation when subjects bargain over abstract situations (Hyder et al, 2000). In future research it may be interesting to analyze the relative performance of the PM and NEG in specific bargaining situations. This is out of the scope of the present paper.

After all subjects casted their votes or all subjects reached an agreement (or exhausted their negotiation time), the program computed the payoff of each subject and displayed: (i) his/her valuations; (ii) the outcomes; and (iii) his/her payoff for that period. When subjects used the PM, the following information was also provided: (iv) his/her vote; and (v) his/her opponent's vote.

As noted in Table 1 below, the sessions with the PM consisted of many more periods than the NEG ones. The number of periods was determined so that each session approximately took an hour and a half. When parties negotiated, they took much longer to reach a decision so we reduced the number of periods. In the present paper we only look at the 18 initial periods of the PM sessions so that we can compare the use of different mechanisms while keeping constant the experience of our subjects. ${ }^{12}$ The table below summarizes all our sessions.

\begin{tabular}{ccccccc} 
Mechanism & Information & Sessions & $\begin{array}{c}\text { \# Independent } \\
\text { Groups }\end{array}$ & Subjects & Periods & $\begin{array}{c}\text { Time } \\
\text { Constraint }\end{array}$ \\
\hline Negotiation & Complete & 2 & 6 & 18 & 18 & 4 minutes \\
\hline Negotiation & Incomplete & 4 & 5 & 20 & 18 & 4 minutes \\
\hline Point Mechanism & Complete & 2 & 6 & 18 & $50(18)^{*}$ & - \\
\hline Point Mechanism & Incomplete & 3 & 9 & 18 & $50(18)^{*}$ & - \\
\hline
\end{tabular}

Table 1: Details of all our experimental sessions

* the PM sessions had 50 periods but in this paper we restrict our analysis to the first 18 periods

\footnotetext{
${ }^{10}$ For example $(300,200,100),(100,400,100),(100,100,400)$ or $(500,50,50)$ were all equally likely.

${ }^{11}$ Framing effects imply that voters may behave differently when they are assigned payments $(1,2)$ or $(200,400)-$ see the seminal reference Kahneman and Tversky (1983).

${ }^{12}$ An extensive analysis of the PM sessions can be found in Hortala-Vallve and Llorente-Saguer (2010).
} 


\subsection{Procedures}

In total 206 subjects participated in 11 sessions with 18 to 20 subjects in each session. No subject participated in more than one session. Students were recruited through the online recruitment system ORSEE (Greiner, 2004) and the experiment took place on networked personal computers in the LEEX at Universitat Pompeu Fabra between April 2006 and November 2011. The experiment was programmed and conducted with the software z-Tree (Fischbacher, 2007). The data and program code for the experiment are available upon request.

The same procedure was used in all sessions. Instructions (see Appendix) were read aloud and questions answered in private. Students were asked to answer a questionnaire to check their full understanding of the experimental design (if any of their answers was wrong the experimenter referred privately to the section of the instructions where the correct answer was provided). Students were isolated and could only communicate through the computer terminals.

The final payment of the session was computed by adding the payoff obtained in three (randomly selected) periods. Additionally subjects received a show up fee of 3 Euros. At the end of each session participants were asked to fill in a questionnaire on the computer and were given their final payment in private. Session length, including waiting time and payment, was around an hour and a half. The average payment was $€ 14.34$.

\section{Aggregate Results}

Allowing negotiating parties to settle their dispute is a difficult task. Inefficiencies arise because parties do not extract gains from trade inherent in their different attitudes to risk, patience or relative intensities across different policy dimensions. Strict Pareto improvements fail to be realized due to bounded rationality, the strategic behavior of the involved parties or due to the lack of complete information. In this paper, we restrict the possibility of disagreement to the differing relative intensities among the various decisions and analyze the role of information about these intensities under two different bargaining protocols.

In this paper we compare both mechanisms PM and NEG under two informational settings using three different welfare measures. First, we consider the utilitarian measure by computing the average payoff obtained by our subjects. Second, we normalize the utilitarian measure by considering the welfare gains over the minimum average welfare the two matched subjects could attain (and normalizing by the maximal possible difference in welfare). That is, given the subjects' joint payoff $\mathrm{W}$, we define the score achieved by each pair of subjects as score $=\frac{W-\operatorname{Min} W}{\operatorname{Max} W-\operatorname{Min} W}$, where MinW and MaxW are the minimum and maximum joint welfare the pair of subjects could possibly achieve in the particular situation. Third, we calculate the percentage of situations in which our subjects reached Pareto optimal payoffs across all situations. 
Table 2 below summarizes the three different measures for each of our four treatments. The message is the same regardless of the welfare measure we consider. Firstly, information has opposite effects in each bargaining protocol. When subjects negotiate, they achieve better outcomes when information about both bargaining parties is common knowledge. Instead, when subjects use the PM, subjects' welfare is superior when each bargaining party knows his/her own preferences and only knows the prior distribution from which his/her opponent's preferences are drawn. Using the average/percentage for each of our independent observations, the Mann-Whitney test supports that all these differences are significant at a $10 \%$ level except for the comparison of average payoffs in the case of negotiation.

Secondly, when we do not change the informational setting, negotiation does better than the PM when information is complete. However, the opposite is true when information is incomplete. Once again, all these differences are statistically significant (at the 5\% level) according to the Mann-Whitney test.

\begin{tabular}{cccc} 
& & \multicolumn{2}{c}{ Information } \\
\cline { 3 - 4 } & & Complete & Incomplete \\
\hline \multirow{3}{*}{ Negotiation } & Average Payoff & 383.06 & 373.52 \\
& Average Score & 87.24 & 83.29 \\
& \% Pareto efficient outcomes & 84.57 & 77.22 \\
\hline \multirow{2}{*}{ Point } & Average Payoff & 369.53 & 385.79 \\
Mechanism & Average Score & 81.87 & 86.52 \\
& \% Pareto efficient outcomes & 75.93 & 84.16 \\
\hline
\end{tabular}

Table 2: Average Payoff, Average Score and Percentage of Pareto efficient outcomes in each treatment.

The averages reported above are indicative of the differing role of information in unconstrained negotiation or PM. However, by aggregating all our data we are not taking into account the different bargaining situations we induced. Recall that subjects' payments were uniformly distributed in the set of payments that are multiples of 50 and add up to 600. This implies that in some situations our subjects faced a strategic situation similar to one of a zero-sum game where gains for one party are losses for the other (this occurs when both subjects have the exact same payments for each of the three issues). Instead, in some situations subjects faced a strategic situation more akin to a coordination game where there are obvious gains from trade. In order to capture the different strategic situations subjects faced we use the classical measure of conflict by Axelrod (1967): the conflict of interest (CI). By measuring the inherent gains from trade we are able to summarize the strategic situation our subjects faced. CI takes a value of one, when subjects are in a zero-sum game (maximal conflict) and takes value zero when there is no conflict, i.e. subjects need to coordinate on the Pareto superior outcome. In the Appendix we explicitly construct this measure by directly adapting the Axelrod's measure to our setting. 
In Figure 1 below we display the three efficiency measures achieved by each mechanism under complete or incomplete information disaggregated by the level of conflict. Each figure compares an efficiency measure in a particular bargaining protocol given complete or incomplete information: in gray we display the minimum of the respective variable achieved by either complete or incomplete information; in color, we display the excess above the minimum of the relevant informational setting. This allows visualizing whether each mechanism does best under complete or incomplete information.

The first aspect worth emphasizing in Figure 1 is that the index of conflict is highly correlated with the three measures of efficiency. The correlation with average payoff is due to the fact that the index of conflict captures an upper bound in the possible welfare gains. A bit more surprising is the positive correlation with the index and the average score and the percentage of Pareto efficient situations. This emphasizes that having a high index of conflict not only establishes an upper bound on the possibilities of trade but also makes any improvement with respect to the minimum achievable welfare more difficult to attain.

Most importantly, Figure 1 tells us that the main message from Table 2 is preserved across different levels of conflict. At any level of conflict, subjects negotiating do better in a complete information situation than in an incomplete information situation (all bars but one in the left figure are grey and white), but more so for high level of conflict as compared to low level of conflict. Instead, the opposite occurs when subjects used the PM in almost all conflict levels and especially for low levels of conflict, subjects using the PM do better when information is incomplete.

In Figure 2 we rearrange the same information so as to emphasize which mechanism does best in each informational setting. Once again we observe that regardless of the level of conflict, negotiation does better than PM when information is complete for any level of conflict. When information is incomplete we see that PM does better especially at low levels of conflict. ${ }^{13}$

In many real world situations it is not easy to reveal the available information (truthfully) to the bargaining parties. In such situations, Figure 2 is indicative of which mechanism should be used in each case: when bargaining parties know the preferences of their opponent, unconstrained negotiation is best; instead, in a situation in which not much is known about the opponent's preferences both bargaining parties are better off by using PM (or any related mechanism).

In what follows we dissect the aggregate comparison above to understand the source of the observed differences. We start by looking at the role of information with each of the two bargaining protocols. We build all our analysis on the payoff each bargaining pair achieves -analogous qualitative results obtain when we consider the average score and the probability of reaching a Pareto efficient allocation (the correlation between the payoff and the score and Pareto efficiency are .41 and .51 respectively; when we consider the joint payoff achieved by each pair of subjects the correlations raise to .59 and .73 ).

\footnotetext{
${ }^{13}$ As one can guess from the graph, not all these differences are significantly different from zero. About one fourth of the comparisons are significant.
} 

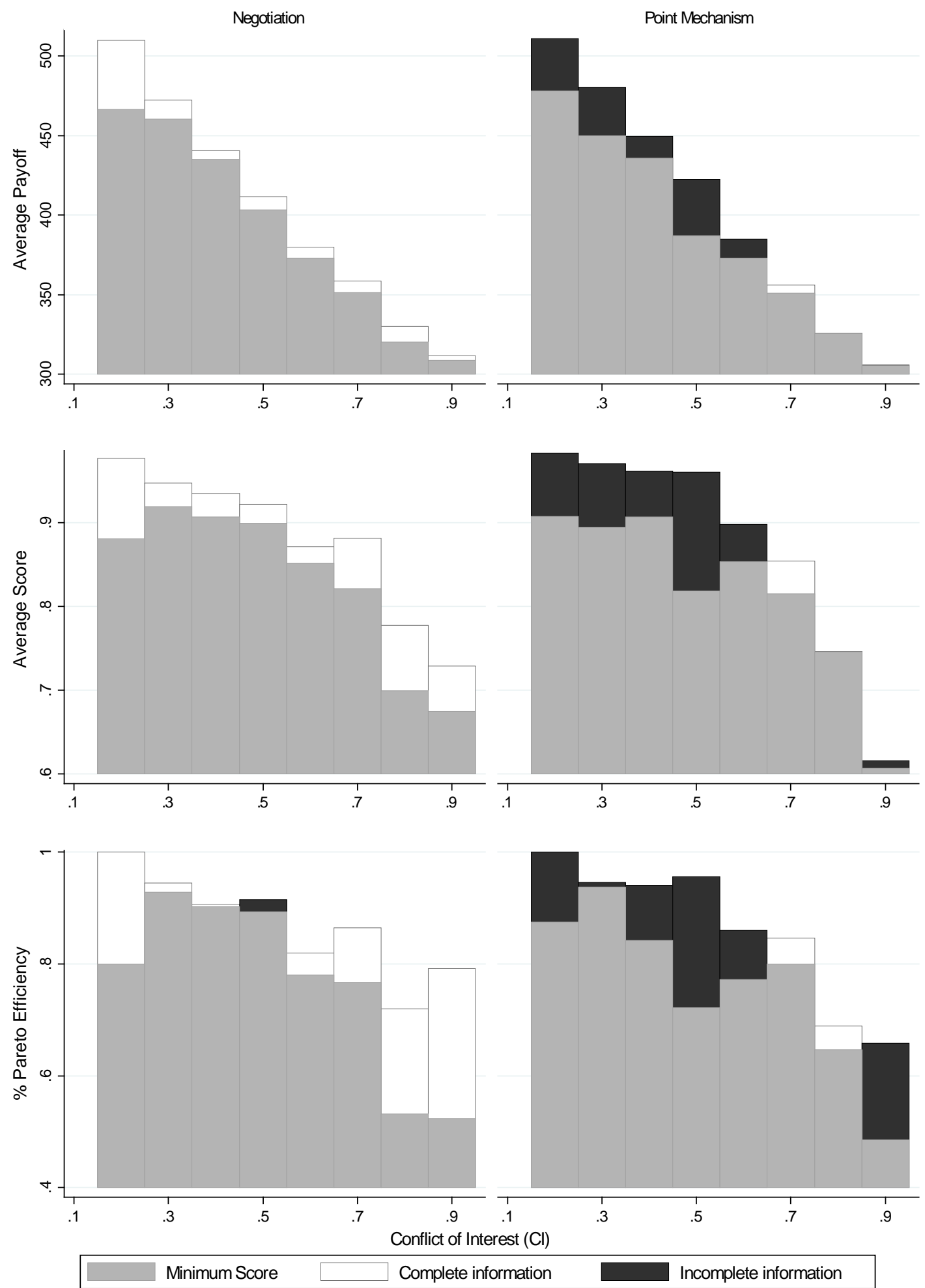

Figure 1: Role of information in each bargaining protocol by the Conflict of Interest. The figures on the left compare the bargaining protocol negotiation under complete and incomplete information; the ones on the right do the same with the PM. The first row of figures displays the average payoff, the second row the average score and the third row the percentage of Pareto efficient situations. In gray we display the minimum of the respective variable achieved by either complete or incomplete information. Above these bars we display the excess above the minimum of the relevant informational setting: in white when the treatment that achieves the highest efficiency is the treatment with complete information; in black when it is the treatment with incomplete information. 

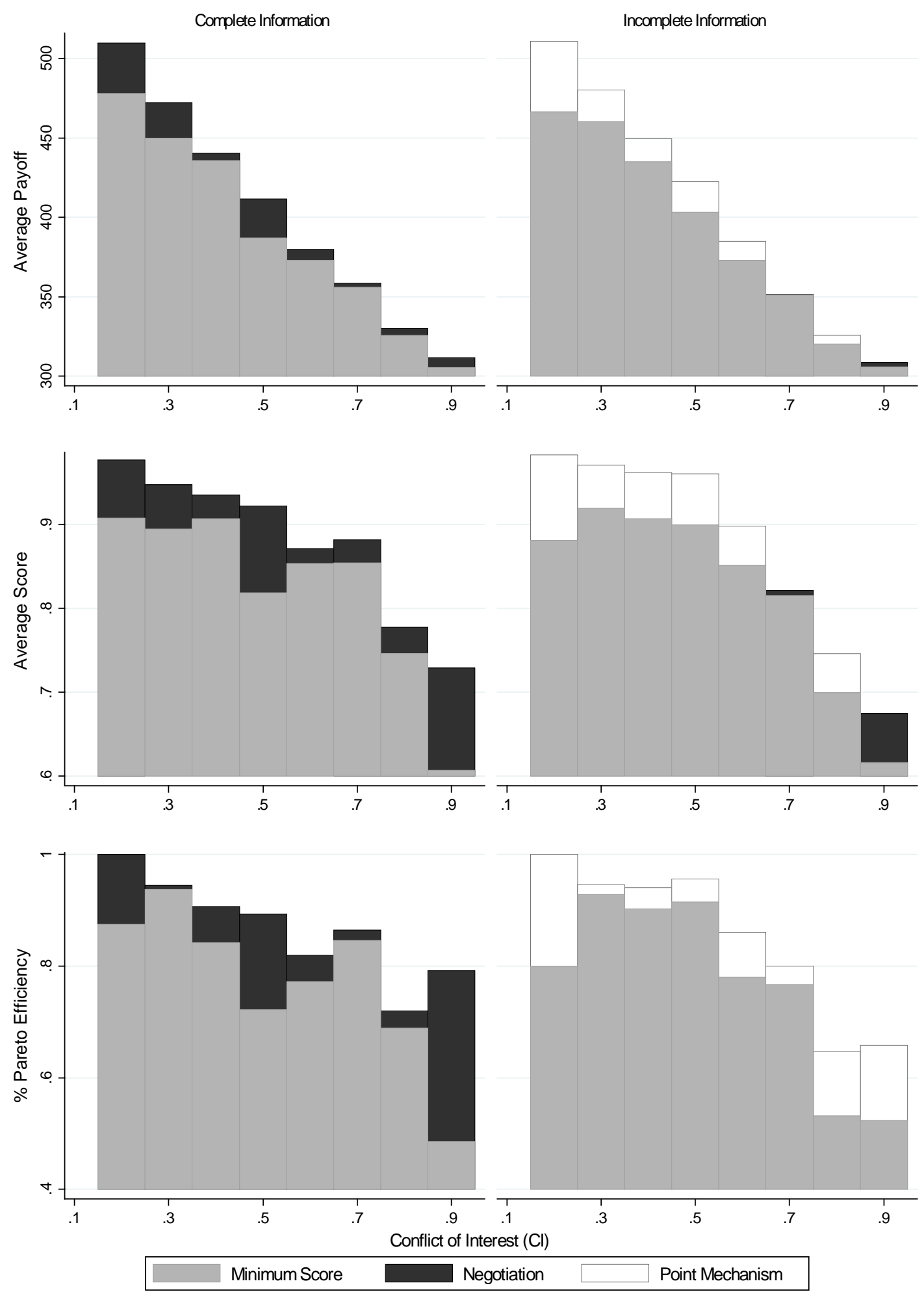

Figure 2: Role of bargaining protocol in each informational setting by the Conflict of Interest. The figures on the left compare the situation with complete information when the bargaining protocol is negotiation or PM; the ones on the right do the same with incomplete information. The first row of figures displays the average payoff, the second row the average score and the third row the percentage of Pareto efficient situations. In gray we display the minimum score achieved by either negotiation or PM. Above these bars we display the excess above the minimum score of the relevant mechanism: in black when the mechanism that achieves the highest average score is negotiation and in white when it is the PM. 


\section{The Role of Information}

The key to understand the role of information in each of our two mechanisms is to understand the different incentives to play strategically under each bargaining protocol. We now present suggestive evidence in support of the hypothesis that when subjects use the PM, their incentives to bluff or strategize are most prevalent when information is complete; when subjects negotiate this happens when information is incomplete.

\subsection{Point Mechanism}

In previous work (Hortala-Vallve and Llorente-Saguer 2010, 2012) we characterize the equilibria in pure strategies of the bargaining protocol PM under complete and incomplete information. When information is incomplete, the equilibrium is very intuitive having parties invest more points in issues that are more valued (i.e. equilibrium strategies are monotone). ${ }^{14}$ Our subjects’ payoff is very close to the one predicted by theory even when subjects only replicated equilibrium play $25.21 \%$ of the times. In Hortala-Vallve and LlorenteSaguer (2010) we analyze the source of these surprising results and see that subjects reap most efficiency gains by playing monotone strategies. When looking at our PM treatment with incomplete information we observe that $91.36 \%$ of the actions played are monotone. We can thus explain the good performance of the PM under incomplete information by the use of such intuitive strategies.

When information is complete, subjects have more incentives to strategize: they know their own valuations as well as the opponent's ones so they can form expectations on the way the opponent will play and try to best respond to such expected plan of action. In Hortala-Vallve and Llorente-Saguer (2012), we show that under complete information an equilibrium in pure strategies exists when both subjects' preferred issue coincides (and its valuation is above the sum of valuations of the other issues for each subject): in equilibrium, both players invest all of their points in their preferred issue and ties occur in all issues. There is another set of situations in which an equilibrium in pure strategies exists: when the preferred issue of each party is the least preferred issue of the opponent. The equilibrium in this case is such that both subjects win their most preferred issue and tie their second most preferred one. ${ }^{15}$ In $28.70 \%$ of our matched pairs subjects faced a situation in which an equilibrium in pure strategies existed (the equilibria in mixed strategies have not been fully characterized). Among the pure equilibrium situations, around a fourth of our observations coincide with equilibrium strategies (27.96\%) and 93.52\% are indeed monotonic.

\footnotetext{
${ }^{14}$ See the appendix for a detailed description of the equilibrium

${ }^{15}$ See the appendix for a detailed description of the equilibria.
} 
The key difference between the setting with complete and incomplete information is the presence of monotone strategies. While with incomplete information we observed $91.36 \%$ of monotone strategies, now with complete information we only observe $66.51 \%$. Regressions in Table 3 show that this different pattern can explain the differences of payoffs in these two treatments. This table reports Random effects GLS regressions of the payoff, clustered by independent groups (as we do for the rest of regressions). In Regression 1, we regress the payoff achieved by each subject on a dummy variable that takes value one when information is complete (while controlling for the level of conflict). We see that the coefficient for the dummy variable is highly significant and negative. In other words, in line with the results presented in the previous section, when information is complete subjects reach a lower payoff. However, when in Regression 2 we include a dummy variable that takes value one when the player plays monotone strategies (variable Monotone Strategies), the previous result no longer holds true: the coefficient on having complete information is not significantly different from zero. In other words, the negative impact of complete information on the score occurs through the use of monotone strategies. Finally, we also include a regression where we control for the situations in which both subjects played a monotone strategy. The result of this specification is similar to the previous, but the latter variable absorbs part of the effect. In section 5.3 we will discuss further the strategizing effect for the case of the PM with complete information.

\begin{tabular}{lccc} 
& Regression 1 & Regression 2 & Regression 3 \\
\hline Complete Information & -10.775 & -1.101 & -0.121 \\
& $(2.968)^{* * *}$ & $(3.402)$ & $(2.920)$ \\
Index of Conflict & -280.179 & -280.765 & -280.851 \\
& $(10.949)^{* * *}$ & $(9.396)^{* * *}$ & $(9.026)^{* * *}$ \\
Period & 0.631 & 0.468 & 0.453 \\
& $(0.332)^{* * *}$ & $(0.269)^{*}$ & $(0.282)^{* * *}$ \\
Monotone Strategies & & 38.887 & 35.791 \\
& & $(9.131)^{* * *}$ & $(14.468)^{* *}$ \\
Monotone Strategies by Both & & & 4.301 \\
& & & $(8.538)$ \\
Constant & 550.201 & 516.571 & 516.011 \\
& $(6.755)^{* * *}$ & $(8.753)^{* * *}$ & $(8.008)^{* * *}$ \\
\hline Observations & 1620 & 1620 & 1620 \\
R-squared & 0.25 & 0.27 & 0.27
\end{tabular}

Table 3: Random effects GLS regression of the payoff obtained with Point Mechanism. Hubbard-White robust standard errors in parentheses, clustered at the independent group level in brackets. ${ }^{* * *}, * *$ and $*$ denote significance at $1 \%, 5 \%$ and $10 \%$ levels, respectively. 


\subsection{Negotiation}

The classical work on the role of information in bargaining is Roth and Murnighan (1982). In their setup, subjects bargain on how to distribute lottery tickets to win a certain prize. This prize is usually different for each of the bargaining parties and the authors vary the information available to each bargaining party about the opponent's prize. Subjects can send proposals and non-binding messages. The key result is that when information is not common knowledge, subjects tend to agree on egalitarian outcomes in the sense that they both get a similar number of lottery tickets. Instead, when information is complete, subjects tend to distribute lottery tickets so as to equate the expected payoff to each party. We can draw a clear parallelism with our findings in the negotiation treatments. ${ }^{16}$

In the presence of incomplete information, subjects are not sure of the valuations of the other subject so the natural fairest norm is that each party wins the 'same' number of issues. In other words, the outcome should be a tie in all issues (this is also the outcome in case of disagreement) or each party winning an issue and a tie occurring on the third one. Indeed we observe significantly more fair outcomes when information is incomplete (68.89\% compared to $61.73 \%$ with complete information -Mann-Whitney, $\mathrm{z}=1.78, \mathrm{p}=0.0755$ ).

\begin{tabular}{lccc} 
& & \multicolumn{2}{c}{$\begin{array}{c}\text { excluding negotiating outcomes } \\
\text { where subjects reach the } \\
\text { status quo outcome }\end{array}$} \\
\cline { 3 - 4 } & Regression 1 & Regression 2 & Regression 3 \\
\hline Complete Information & 7.6 & 4.744 & 4.347 \\
& $(3.630)^{* *}$ & $(1.474)^{* * *}$ & $(1.639)^{* * *}$ \\
Index of Conflict & -260.775 & -263.83 & -263.394 \\
Period & $(12.414)^{* * *}$ & $(6.389)^{* * *}$ & $(5.999)^{* * *}$ \\
& 0.222 & 0.605 & 0.569 \\
Win-Draw-Lose & $(0.164)$ & $(0.123)^{* * *}$ & $(0.120)^{* * *}$ \\
& & & -7.875 \\
Constant & & & $(3.014)^{* * *}$ \\
& 532.41 & 541.45 & 546.31 \\
Observations & $(9.109)^{* * *}$ & $(4.541)^{* * *}$ & $(5.499)^{* * *}$ \\
R-squared & 2088 & 1702 & 1702 \\
& 0.39 & 0.38 & 0.38
\end{tabular}

Table 4: Random effects GLS regression of the payoff obtained with Negotiation. HubbardWhite robust standard errors in parentheses, clustered at the independent group level in brackets. The variable Win-Draw-Lose is a dummy variable that takes a value of 1 when each subject in the matched pair wins an issue and the third issue is tied. ${ }^{* * *},{ }^{* *}$ and $*$ denote significance at $1 \%, 5 \%$ and $10 \%$ levels respectively.

\footnotetext{
${ }^{16}$ Roth and Murnighan (1982) study the case in which information is asymmetric in the sense that only one player is better informed than the other player. These cases are not discussed here.
} 
Regression 1 in Table 4 regresses the payoff achieved by each subject in Negotiation treatments on only a dummy variable for the level information (while controlling by the level of conflict). The coefficient on having complete information is significant and positive. In other words, in line with the results presented in the previous section, subjects in complete information situations reach a higher payoff. Later in regressions 2 and 3 we analyze the impact of not reaching an agreement and implement the outcome where all issues are tied (i.e. reaching the status quo outcome). Note that this outcome uniquely determines the payoff of both subjects (300) so a dummy that takes value 1 when such outcome was achieved cannot be included in the first specification. It is for this reason that in regressions 2 and 3 we look at the subsample of observations where we exclude the observations in which subjects reach the status quo outcome (we disregard whether this happens by reaching the time limit or whether this outcome is agreed prior to the time limit; looking at this two separate cases does not vary our results). Regression 2 is analogous to regression 1 but with the subsample of observations. We see that the coefficient of the dummy that captures the presence of complete information is still significant, but the magnitude of the effect decreases considerably. Finally, in regression 3 we add a dummy variable capturing whether subjects reached a fair outcome different than the status quo outcome in which each subject wins one issue and the third issue is tied. We see that, in line with the findings in Roth and Murnighan (1982), coordinating in such outcomes has a negative impact on overall payoff.

There is another aspect that differentiates our setting from the one in Roth and Murnighan (1982). In their setting any concession by one bargaining party is a victory by the opponent (maximal conflict of interest). However, in our setting there are many allocations that are not Pareto efficient. In the presence of complete information, both subjects have enough information to avoid Pareto suboptimal outcomes. Instead, when information is incomplete, subjects strategize and try hiding their true valuations so as to obtain a better final outcome. This implies that Pareto suboptimal outcomes may occur due to the misreporting of information and the inherent lack of trust in the opponent's declarations. It is indeed the case that under complete information we observe less Pareto efficient allocations and this is also a key factor in the better performance of the complete information treatment.

A different way to show that under incomplete information negotiating parties are less likely to obtain gains from trade is by looking at the percentage of observations in which both subjects implemented the status quo outcome in which all issues are tied. When information is complete, this outcome is reached $15 \%$ of the times ( $9 \%$ due to reaching the time limit); instead, when information is incomplete subjects reach this outcome more often: $21 \%$ (18\% due to reaching the time limit). 


\subsection{The role of "strategizing" and decision making time}

Heuristically we could say that information plays a very different role with each of the mechanisms due to the subjects' incentives to strategize: the presence or lack of information induces very different strategic incentives under each bargaining protocol. When information is incomplete subjects cannot do much strategizing when using the PM: subjects only know their preferences so the best they can do is using monotone strategies so as to increase the probability to win the issues they value most. Instead, when information is complete subjects can form expectations on the opponent's optimal plan of action and best respond to it. Consider for instance one of our observations in which two subjects had preferences $(300,250,50)$ and $(150,50,400)$. The first subject played a monotone strategy and distributed points in the following way: $(3,2,1)$. The second subject instead played $(4,0,2)$-indeed a non-monotone strategy which is a best response to the action played by the first subject. ${ }^{17}$ Obviously, this strategy combination is not an equilibrium.

In the negotiation treatments, having information about the opponent's preferences reduces the role for strategizing, as subjects cannot try to reach a better outcome by misrepresenting their preferences.

As a suggestive measure of the role of strategizing we look at the time subjects needed to reach a decision or the time they spent to distribute their points across the three issues. Longer times could be associated with subjects being more likely to devise the best plan of action or longer negotiation with the opponent. This is in line with the findings in Rubinstein (2007), where it is shown that response times requiring the use of cognitive reasoning are associated with higher response times.

Complete Incomplete

\begin{tabular}{cccc}
\multirow{2}{*}{ Negotiation } & Average & 119 & 162 \\
& St. Dev. & 79 & 74 \\
\hline \multirow{2}{*}{ Point Mechanism } & Average & 28 & 16 \\
& St. Dev. & 12 & 10 \\
\hline
\end{tabular}

Table 5: Average time and standard deviation needed to reach an agreement (in seconds). Recall that negotiation sessions had a time constraint of 240 seconds.

Subjects in the negotiation sessions spend more time to reach a decision -this is a natural consequence of subjects not needing to reach an agreement when using the PM. More importantly, we observe that when

\footnotetext{
${ }^{17}$ One could argue that this player is best responding to his opponent bidding truthfully (i.e. matching the ratio of points to his relative intensities). This could be interpreted as level 0 behaviour according to cognitive hierarchy models (see Nagel 1995).
} 
subjects negotiate they need more time to reach an agreement when information is incomplete. Instead, subjects using the PM need more time when information is complete. ${ }^{18}$

\section{The Role of the Bargaining Protocol}

The previous analysis shows the different role of information in different bargaining protocols. However, in many circumstances the amount of available information cannot be easily changed. In these circumstances it is useful to look at the orthogonal comparison in which we do not change the informational setting and check which mechanism does best.

\subsection{Incomplete Information}

The key question when comparing these mechanisms is whether a mechanism that asks parties to move simultaneously can resolve disputes as well as unconstrained negotiation. We observe that optimal behavior under the PM mechanism with incomplete information is indeed followed by our subjects: 91\% play monotone strategies by investing more votes in those issues that are valued more. This allows the gains from trade from the subjects' differing relative valuation to be extracted. Instead, when subjects freely negotiate they have incentives to misrepresent their preferences so as to obtain a better outcome (what we just called 'strategizing'). This strategic behavior pushes subjects away from the utilitarian first best. Moreover, in situations with a high CI, negotiating subjects do not realize there are gains from trade and focus their discussion instead on a single divisive issue. This implies that unconstrained negotiation yields many more disagreements; in which case all issues are tied. Instead, when using the PM subjects can extract gains from their (possibly) small differences in their relative valuations by using monotone strategies.

The subjects' score in the PM treatments is significantly higher than their payoff in the negotiation treatments. The two regressions in table 6 show that these differences are mainly explained by the situations in which gridlock occurs, i.e. subjects do not reach an agreement or implement the outcome that would have been implemented in that case. The difference between these two regressions is that in the first one we look at the whole sample of observations with incomplete information but in the second regression we drop the observations in which subjects reached the 'no agreement' outcome (we disregard whether this happens by

18 All the aforementioned differences are significant at the 1\% level (Mann Whitney test) with the only exception of difference between the time in the negotiation sessions with complete information and incomplete information that is marginally insignificant $(z=1.643, p=0.1003)$. However, this difference becomes significant at the $10 \%$ if we drop the initial six periods of the data (out of 18 periods). In the appendix we include Figure A3 in which we report average times in each of our treatments by the level of conflict. 
excluding outcomes where

subjects reach the

status quo outcome

\begin{tabular}{lcc}
\hline & Regression 1 & Regression 2 \\
\hline Negotiation & -9.773 & -0.79 \\
& $(3.391)^{* * *}$ & $(2.526)$ \\
Index of Conflict & -277.574 & -280.272 \\
& $(12.546)^{* * *}$ & $(8.053)^{* * *}$ \\
Period & 0.455 & 0.736 \\
& $(0.246)^{*}$ & $(0.220)^{* * *}$ \\
Constant & 550.298 & 550.604 \\
& $(6.710)^{* * *}$ & $(5.261)^{* * *}$ \\
\hline Observations & 2412 & 2074 \\
R-squared & 0.31 & 0.29
\end{tabular}

Table 6: Random effects GLS regression of the payoff obtained with Incomplete Information. Hubbard-White robust standard errors in parentheses, clustered at the independent group level in brackets. ***, $* *$ and $*$ denote significance at $1 \%, 5 \%$ and $10 \%$ levels respectively.

reaching the time limit or whether this outcome is agreed prior to the time limit). We see that the negative coefficient capturing whether subjects negotiated is negative in regression 1 but becomes positive in regression 2 when we reduce our sample of observations. In other words, the negative performance of negotiation vis-à-vis the PM is driven by the inability of bargaining parties to avoid gridlock and extract gains from trade.

\subsection{Complete Information}

We hinted above that when information is complete subjects have incentives to play strategically when using the PM but only need to coordinate on the best possible outcome when negotiating. The success of negotiation when information is complete is mainly due to two factors: knowing everyone's valuations dissipates the role for strategizing and bluffing about one's preferences and, very importantly too, when subjects negotiate we introduce the possibility of renegotiation thus increasing the possibility of avoiding Pareto suboptimal allocations. Actually, the PM treatment with Complete Information and Negotiation with Incomplete Information can be considered situations in which only one of these elements is at play. ${ }^{19}$

\footnotetext{
${ }^{19}$ We are indebted to Rachel Croson for bringing this fact to our attention.
} 
In table 7 below we replicate the information in table 2 about Pareto efficiency. When looking at the first aspect about knowing each other's preferences we can see that moving from negotiating with complete information to negotiating with incomplete information efficiency levels drop from $84.57 \%$ to $77.22 \%$. Instead, moving from a situation in which subjects can renegotiate any outcome to a situation in which this renegotiation is not present (moving from NEG to the PM when information is complete) drops efficiency from $84.57 \%$ to $75.93 \%$. Overall we can see that the worst performer is the PM under complete information.

\begin{tabular}{ccc} 
& Complete & Incomplete \\
\hline Negotiation & $84.57 \%$ & $77.22 \%$ \\
\hline Point Mechanism & $75.93 \%$ & $84.16 \%$ \\
\hline
\end{tabular}

Table 7: Percentage of Pareto efficient allocations in each of our four treatments.

Looking at Pareto optimal allocations may hide away egalitarian concerns as subjects may reach outcomes that are not Pareto optimal but the Pareto superior one may benefit only one bargaining party. On this vein we check the robustness of the previous finding by looking at strict Pareto optimal allocations (i.e. situations in which both players cannot simultaneously be better off) and find our previous results unchanged: subjects in the PM treatment under complete information are still the ones that obtain the lowest percentage in strict Pareto optimal allocations.

\section{Conclusion}

This paper is a first attempt to build a bridge between two related areas of research. We have analyzed the performance of a Point Mechanism and unconstrained Negotiation in a controlled laboratory experiment. The first bargaining protocol is similar to a family of mechanisms analyzed in voting and the social choice literature such as Adjusted Winner (Brams and Taylor, 1996 \& 1999), Storable Votes (Casella, 2005), Qualitative Voting (Hortala-Vallve, forthcoming) or, more generally, any linking mechanism (Jackson and Sonnenschein, 2007). The essence of the PM is that it allows parties to unilaterally trade-off their interests across dimensions and strengthens their position on those issues they mostly care about. It follows that the comparison between these mechanisms and unconstrained negotiation should parallel the findings in this paper. The second bargaining protocol, which we just call Negotiation, represents the usual way parties resolve their disputes.

The key to the comparison between these mechanisms has been the level of information parties have about their opponents' preferences. We have looked at two extreme cases: one in which subjects perfectly know the 
valuations of their opponent, and another one in which they only have a flat prior about their opponent's valuations. We have seen that information plays an important role in the comparison between these mechanisms. Having complete information allows subjects to strategize when using the PM but instead diminishes the possibility of strategizing when subjects negotiate. Overall, we have shown that the possibility of strategizing harms overall welfare. Looking at the same data from a different perspective, we have asked ourselves what would be the ideal bargaining protocol given a particular informational situation. We have seen that the PM outperforms unconstrained negotiation when information is incomplete and the opposite occurs when information about preferences is common knowledge. An interesting avenue of future research is analyzing the role of asymmetric information. That is, what would happen when only one of the two bargaining parties knows the valuation of the opponent just like the work by Roth and Murnighan (1982) or Mitzkewitz and Nagel (1993). Similarly, in the future it may be interesting to introduce the possibility of renegotiation when using the PM mechanism -note that renegotiation is possible by design when the bargaining protocol is unconstrained negotiation.

Finally, it is worth noting that we have restricted our attention to unconstrained negotiation through computer terminals in order to avoid the unquantifiable effects of face-to-face interaction. How our results extend to such situations remains an open question. While there is consensus in the literature that negotiation through chat might take more time than face-to-face negotiation (see for instance Purdy et al, 2000) there is no consensus about whether negotiation through chat obtains better or worse outcomes than face-to-face negotiation. Previous authors have shown that written communication (as compared to oral communication) may ease reaching an agreement (see Croson, 1999). On this line, Carnevale et al (1981 and 1986) show that denying visual access to the negotiation parties achieves more integrative results. Instead, Purdy and Balakrishnan (2000) compare negotiated outcomes when communication happens face-to-face, through videoconference, through telephone or through computer terminals and find that the latter leads to longer processes and less integrative behavior. 


\section{References}

[1] Axelrod, R. (1967), “Conflict of Interest: An Axiomatic Approach”, Journal of Conflict Resolution, 11, 87-99.

[2] Bazerman, M. and M. Neale (1991), Negotiating Rationally, Free Press, New York.

[3] Bazerman, M. and M. Neale (1983) "Heuristics in Negotiation: limitations to dispute resolution effectiveness," (pp. 51-67) in M. H. Bazerman and R. J. Lewicki (Eds.), Negotiating in organizations, Beverly Hills.

[4] Brams, S. J., and A.D. Taylor (1996), Fair Division: From Cake Cutting to Dispute Resolution, Cambridge University Press

[5] Brams, S. J., and A.D. Taylor (1999), The Win-Win Solution: Guaranteeing Fair Shares to Everybody, New York: Norton.

[6] Bui, T. (1994), “Evaluating Negotiation Support Systems: A Conceptualization”, 27th Annual Hawaii International Conference on System Science, Hawaii.

[7] Camerer, C.F. (2003), "Behavioral Game Theory: Experiments in Strategic Interaction”, Princeton University Press.

[8] Carnevale, P. J. D. and A. M. Isen (1986). "The influence of positive affect and visual access on the discovery of integrative solutions in bilateral Negotiation", Organizational Behavior and Human Decision Processes, 37, 1-13.

[9] Carnevale, P.J.D., D.J. Pruitt and S. Seilheimer (1981), “Looking and competing: Accountability and visual access in integrative bargaining”, Journal of Personality and Social Psychology, 40, 111-120.

[10] Casella, A. (2005), “Storable Votes”, Games and Economic Behavior, 51, 391-419.

[11] Casella, A., A. Gelman and T. Palfrey (2006), “An Experimental Study of Storable Votes”, Games and Economic Behavior, 57, 123-154.

[12] Casella, A., T. Palfrey and R. Riezman (2008), “Minorities and Storable Votes”, Quarterly Journal of Political Science, 3, 165-200.

[13] Casella, A (2011), Storable Votes: Protecting the Minority Voice, Oxford University Press.

[14] Croson, R. (1996), "Information in Ultimatum Games: An Experimental Study," Journal of Economic Behavior and Organization, 30, 197-213. 
[15] Croson, R. (1999), “Look at me when you say that: An Electronic Negotiation Simulation”, Simulation and Gaming, 30, 23-37.

[16] Daniel, T. and J. Parco (2005), "Fair, Efficient and Envy-Free Bargaining: An Experimental Test of the Brams-Taylor Adjusted Winner Mechanism”, Group Decision and Negotiation, 14, 241-264.

[17] Engelmann, D. and V. Grimm (forthcoming), "Mechanisms for Efficient Voting with Private Information about Preferences”, Economic Journal.

[18] Erickson, B., J. G. Holmes, R. Frey, L. Walker and J. Thibaut (1974), "Functions of a third party in the resolution of conflict: The role of a judge in pretrial conferences”, Journal of Personality and Social Psychology, 30, 293-306.

[19] Fischbacher, U (2007), “z-Tree - Zurich Toolbox for Readymade Economic Experiments Experimenter's Manual”, Experimental Economics, 10, 171-178.

[20] Fisher, R., W. Ury, and B. Patton, 1981, Getting to Yes (2nd ed.). New York: Penguin.

[21] Greiner, B. (2004), “The Online Recruitment System ORSEE 2.0 - A Guide for the Organization of Experiments in Economics”, University of Cologne WP Series in Economics 10

[22] Guth, W., R. Schmittberger and B. Schwarze (1982), “An experimental analysis of ultimatum bargaining”, Journal of Economic Behavior and Organization, 3, 367-388

[23] Hart, S. (2006), “Discrete colonel blotto and general lotto games”, International Journal of Game Theory, 36, 441-460.

[24] Holsapple, C. W. and A. B. Whinston (1996), Decision Support Systems: A Knowledge-Based Approach, West Publishing Company.

[25] Hortala-Vallve, R. (forthcoming), “Qualitative Voting”, Journal of Theoretical Politics.

[26] Hortala-Vallve, R. and A. Llorente-Saguer (2010), “A Simple Mechanism to Resolve Conflict”, Games and Economic Behavior, 70, 375-391.

[27] Hortala-Vallve, R. and A. Llorente-Saguer (2012), "Pure-strategy Nash equilibria in non-zero sum Colonel Blotto games”, International Journal of Game Theory, 41, 331-343.

[28] Hyder, E. B., M. J. Prietula \& L. R. Weingart (2000), “Getting to best: Efficiency versus optimality in Negotiation”, Cognitive Science, 24, 169-204.

[29] Jackson, M.O. and H.F. Sonnenschein (2007), "Overcoming Incentive Constraints by Linking Decisions”, Econometrica, 75, 241-257.

[30] Kahneman, D. and A. Tversky (1983), “Choices, values and frames”, American Psychologist, 39, 341350. 
[31] Keeney, R. and H. Raiffa (1991), Negotiation Analysis. University of Michigan Press, Ann Arbor.

[32] Kelley, H. H. (1966). “A classroom study of dilemmas in interpersonal Negotiation”. In K. Archibald (Ed.), Strategic interaction and conflict, Berkeley: University of California, Institute of International Studies.

[33] Kilgour, D. M. (1996). “Negotiation Support Using The Decision-Support System GMCR”, Group Decision and Negotiation, 5(4-6), 371-383.

[34] Mitzkewitz, M. and R. Nagel (1993), "Experimental Results on Ultimatum Games with Incomplete Information”, International Journal of Game Theory, 22, 171-198.

[35] Nagel, R (1995), “Unraveling in Guessing Games: An Experimental Study”, American Economic Review, 85(5), 1313-26.

[36] Raiffa, H. (1982). The art and science of negotiation. Cambridge, MA: Belknap Press of Harvard University Press

[37] Robertson, B. (2006), “The colonel blotto game”, Economic Theory 29, 1-24.

[38] Roth, A. E. (1995), “Bargaining Experiments”, in J. Kagel and A. Roth, eds., Handbook of Experimental Economics, Princeton: Princeton University Press.

[39] Roth, A. E. \& J. K. Murnighan (1982), “The role of information in bargaining: An experimental study”, Econometrica, 50, 1123-1142.

[40] Rubinstein, A. (2007), “Instinctive and Cognitive Reasoning: A Study of Response Times”, Economic Journal, 117, 1243-1259.

[41] Pruitt, D.G. (1981), Negotiation behavior, New York: Academic Press.

[42] Roth, A.E., J.K. Murnighan and F. Schoumaker (1988), “The Deadline Effect in Bargaining: Some Experimental Evidence”, American Economic Review, 78, 806-823.

[43] Schneider, G. and U. Kramer (2004), “The Limitations of Fair Fivision”, Journal of Conflict Resolution, 48, 506-524.

[44] Thompson, L. (2000), The mind and heart of the Negotiator, Prentice Hall, Upper Saddle River.

[45] Yukl, G. A., M. E. Malone, B. Hayslip and T.A. Pamin (1976), “The effects of time pressure and issue settlement order on integrative bargaining”, Sociometry, 39, 277-281. 


\section{Appendix}

\section{A1 A measure of the Conflict of Interest (Axelrod, 1967)}

Given a two-player game, we construct the utility possibility frontier and we denote it $\mathrm{G}(\mathrm{x})$; $\mathrm{G}(\mathrm{x})$ is the maximum utility player 2 can reach when player 1 gets $\mathrm{x}$. This function is defined for $\mathrm{x}$ greater than the minimum possible payoff $(\underline{\pi})$ and smaller than the highest possible payoff $(\bar{\pi})$. However, the games our subjects play only have a finite set of outcomes, thus $\mathrm{G}(\mathrm{x})$ is not defined in all its range. In order to simplify our analysis we convexify the set of outcomes so that the utility possibility frontier is now continuous. Note that convexifying the set of outcomes is analogous to allowing lotteries when subjects have von-Neumann Morgenstern utilities.

A game that allows both players reach their maximum utility simultaneously should be viewed as a game of very little conflict; instead, a game where the gains for one player are losses to his opponent should be viewed as a game of high conflict. The former corresponds to a game of coordination and the latter to a constant sum game. Our measure of conflict captures how close we are from a constant-sum game where no gains from trading issues are possible. This is calculated by computing the area between the maximum utility player 1 can reach and the utility possibility frontier $\mathrm{G}(\mathrm{x})$. Formally it reads as follows:

$$
C I=\frac{2}{(\bar{\pi}-\underline{\pi})^{2}} \int_{\underline{\pi}}^{\bar{\pi}}(\bar{\pi}-G(x)) d x,
$$

where the term before the integral is simply a normalization so that our index is between 0 and 1 . A higher CI indicates that there is a large amount of conflict and a lower CI indicates that there is low level of conflict between the two players.

Below in Figure A1 we depict a situation where player 1 has preferences $(400,50,150)$ and player 2 has preferences (50, 250, 300) -note that $\bar{\pi}=600$ and $\underline{\pi}=0 .{ }^{20}$ The points show the pair of utilities associated to each outcome. For instance, the outcome where player 1 decides on the first issue and player 2 decides on the second and third issues is depicted as the utility pair $(400,550)$. The gray area is, once normalized, the CI of this particular game. This area is bounded above and to the right by the utility possibility frontier of a pure coordination game where both players can achieve the maximum utility thus the conflict is minimum, $\mathrm{CI}=0$; the dashed line indicates utility possibility frontier of a constant sum game where conflict is maximum, $\mathrm{CI}=1$.

\footnotetext{
${ }^{20}$ In the case of negotiation, $\bar{\pi}=600$ and $\underline{\pi}=0$. In the case of the PM the lower and upper bounds change, given that no player can win anything (it can never be the case that a single player has fewer points than his opponent in all issues). In order to make the analysis of both mechanisms comparable, we assume throughout the paper that $\bar{\pi}=600$ and $\underline{\pi}=0$.
} 


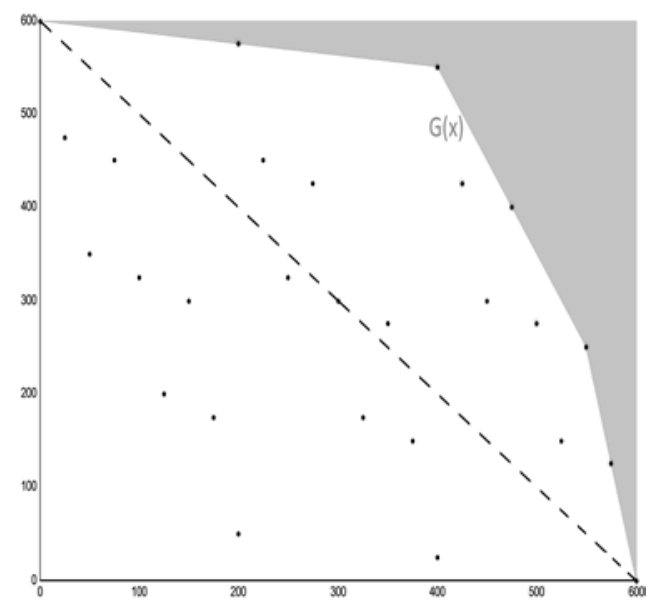

Figure A1: Utility pairs associated to all possible outcomes when two players have preferences (400, $50,150)$ and $(50,250,300) ; \mathrm{G}(\mathrm{x})$ denotes the possibility frontier of the convexified set of outcomes.

Figure A1 shows that CI captures how far the set of utility pairs are from the dashed line where the sum of the subjects' payoffs is constant. However CI fails to capture equitability in the achieved outcomes. Compare for instance a situation where both subjects have preferences $(301,299)$ with a situation where both subjects havepreferences $(599,1)$. Both situations have the same index (CI=1), however the conflict differs. In the first case any subject is almost indifferent between winning any of the two issues (and losing the remaining one). Instead, in the second case both subjects have a strong preference to win the first issue. This simple example highlights the fact that Axelrod's index of conflict captures the possibilities of trade in a 2-player game but remains silent about the inequality among players. Despite its limitation, $\mathrm{CI}$ is extremely helpful in order to characterize different situations by their level of conflict. Below we report the distribution of CI in our experimental sessions (see histograms with relative frequencies and table with the absolute number of observations):

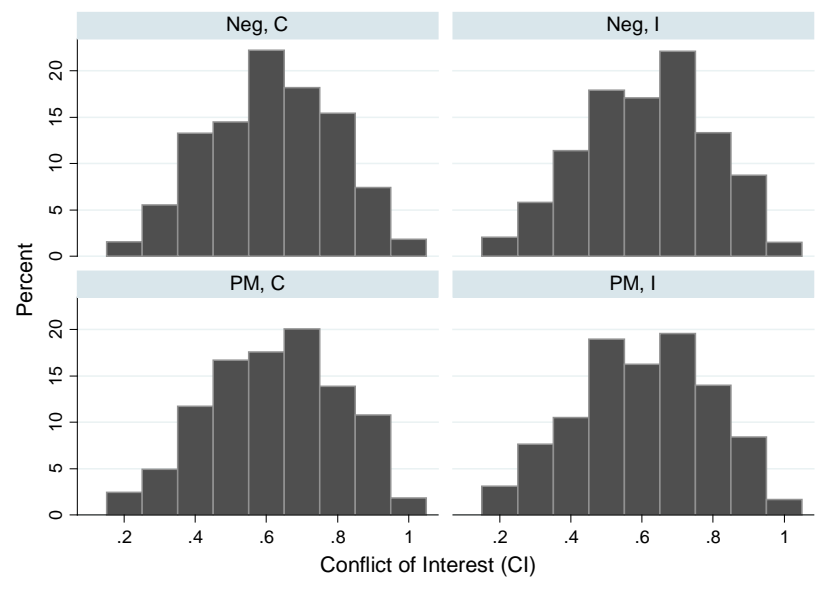

Figure A2: Frequency map of CI in our 4 treatments.

\begin{tabular}{rccccc} 
& \multicolumn{2}{c}{ Neg } & & \multicolumn{2}{c}{ QV } \\
\cline { 2 - 3 } \cline { 5 - 6 } \cline { 5 - 6 } & Complete & Incomplete & & Complete & Incomplete \\
\hline 0.2 & 10 & 30 & & 16 & 30 \\
0.3 & 36 & 84 & & 32 & 74 \\
0.4 & 86 & 164 & & 76 & 102 \\
0.5 & 94 & 258 & & 108 & 184 \\
0.6 & 144 & 246 & & 114 & 158 \\
0.7 & 118 & 318 & & 130 & 190 \\
0.8 & 100 & 192 & & 90 & 136 \\
0.9 & 48 & 126 & & 70 & 82 \\
1 & 12 & 22 & & 12 & 16 \\
\hline Total & 648 & 1,440 & & 648 & 972
\end{tabular}

Table A1: Number of observations for each treatment and each category of conflict used. 


\section{A2 Equilibrium strategies under PM}

The following Table 1 lists all the possible valuation profiles that were induced (ordered in decreasing order) together with the symmetric Bayesian Nash equilibrium action for each type of subject in the incomplete information game (taken from Hortala-Vallve and Llorente-Saguer, 2010):

\begin{tabular}{ccccccc} 
Profile \# & $\boldsymbol{\theta}^{\mathbf{1}}$ & $\boldsymbol{\theta}^{\mathbf{2}}$ & $\boldsymbol{\theta}^{\mathbf{3}}$ & $\mathbf{v}^{\mathbf{1}}$ & $\mathbf{v}^{\mathbf{2}}$ & $\mathbf{v}^{\mathbf{3}}$ \\
\hline 1 & 200 & 200 & 200 & 2 & 2 & 2 \\
2 & 250 & 200 & 150 & 3 & 2 & 1 \\
3 & 250 & 250 & 100 & 3 & 3 & 0 \\
4 & 300 & 150 & 150 & 4 & 1 & 1 \\
5 & 300 & 200 & 100 & 4 & 2 & 0 \\
6 & 300 & 250 & 50 & 3 & 3 & 0 \\
7 & 350 & 150 & 100 & 5 & 1 & 0 \\
8 & 350 & 200 & 50 & 5 & 1 & 0 \\
9 & 400 & 100 & 100 & 6 & 0 & 0 \\
10 & 400 & 150 & 50 & 5 & 1 & 0 \\
11 & 450 & 100 & 50 & 6 & 0 & 0 \\
12 & 500 & 50 & 50 & 6 & 0 & 0 \\
\hline
\end{tabular}

Under incomplete information the equilibrium has very appealing properties that seem intuitive: indifferent subjects (those that care about all the issues with equal intensity) distribute their voting power evenly and extreme subjects concentrate their voting power on their most preferred issue. As explained in the main text, the prescribed strategies are monotonic and this is the key factor in generating outcomes that are close to the efficient ones.

One of the nice features of this setting is that actions can be categorized by level of intensity. Let us define truthful strategy by the voting profile that minimizes the angle with the voting profile. By considering the angle between each voting profile and the voting profile that assigns equal voting power to all issues. This measure captures the degree of intensification of each voting profile or, in other words, the extent to which our subjects concentrate their voting power on a few issues. A feature of the equilibrium is that the equilibrium action is always more intense than the truthful action. Therefore, whenever truthful behavior and equilibrium behavior differ, we can classify all actions as belonging to one of the following 6 (self-explanatory) equivalence classes: $28.10 \%$ of the observations are less intense than truth, $10.79 \%$ coincide with the truthful action, $30.49 \%$ are less intense than theory but more than truthful, 24\% coincide with the equilibrium prediction and $6.52 \%$ play more intense than theory.

The set of games with equilibrium in pure strategies in the case of complete information is generally characterized in Hortala-Vallve and Llorente-Saguer (2012). In the context of the experiment, there exist two potential types of equilibrium. In the first type of equilibrium both voters concentrate all the votes on their preferred issue. This equilibrium can be sustained whenever both subjects' preferred issue coincides and the valuation of this issue is equal or higher than 300 (profile numbers 4-12). In the second type of equilibria, both subjects invest a single vote in their preferred issue and five votes in their second preferred one. This 
equilibrium can be sustained whenever subjects’ preferred issue does not coincide, but the second most preferred issue does and their second valuation must be at least twice their third valuation. Hence, this equilibrium can only be sustained with valuations profiles 3, 5, 6, 8, 10 and 11 . All games that can't sustain these two types of equilibrium have an equilibrium in mixed strategies.

\section{A3 Non-Parametric Tests Summary}

\begin{tabular}{lccc} 
Mechanism & Payoff & Score & Pareto Efficiency \\
\hline Negotiation & 0.1441 & 0.0679 & 0.0541 \\
Point mechanism & 0.0095 & 0.0593 & 0.0386 \\
\hline
\end{tabular}

Table A2: Mann-Whitney test p-values for the comparison between complete and incomplete information. Although not indicated in the table, the p-values for the comparison in the case of negotiation (point mechanism) show that the different variables are significantly higher (lower) for complete information.

\begin{tabular}{lccc} 
Information & Payoff & Score & Pareto Efficiency \\
\hline Complete & 0.0374 & 0.0374 & 0.0364 \\
Incomplete & 0.0136 & 0.0278 & 0.0448 \\
\hline
\end{tabular}

Table A3: Mann-Whitney test p-values for the comparison between Negotiation and the Point Mechanism. Although not indicate in the table, the p-values for the comparison in the case of complete (incomplete) information show that the different variables are significantly higher (lower) for Negotiation.

\section{A4 Time needed to reach an agreement}

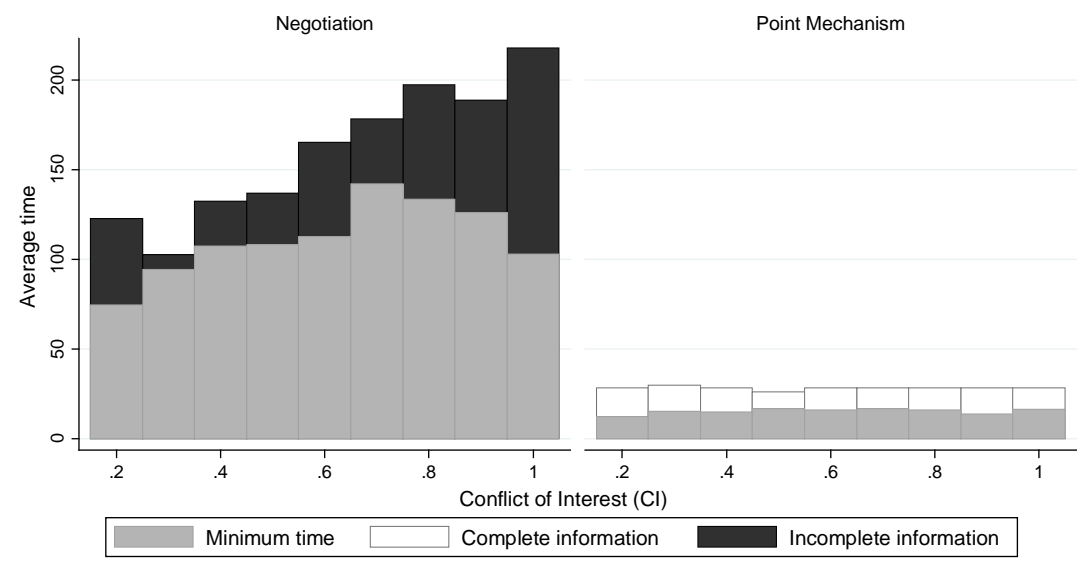

Figure A4: Average time of agreement by the Conflict of Interest. The left figure compares the bargaining protocol negotiation under complete and incomplete information; the right figure does the same with the PM. In gray we display the minimum of the average time achieved by either complete or incomplete information. Above these bars we display the excess above the minimum of the relevant informational setting: in white when the average time used in complete information is higher; in black when the time with incomplete information is higher. 